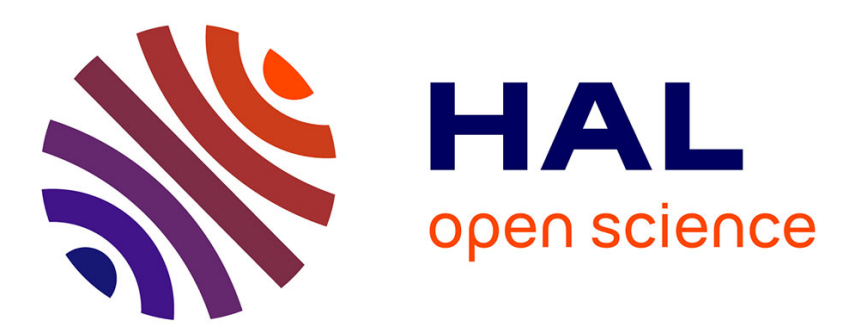

\title{
A combined thermo-mechanical model for Tribological Surface Transformations
}

Grégory Antoni, Thierry Désoyer, Frédéric Lebon

\section{To cite this version:}

Grégory Antoni, Thierry Désoyer, Frédéric Lebon. A combined thermo-mechanical model for Tribological Surface Transformations. Mechanics of Materials, 2012, 49, pp.92-99. 10.1016/j.mechmat.2011.12.005 . hal-00662531

\section{HAL Id: hal-00662531 https://hal.science/hal-00662531}

Submitted on 14 Mar 2018

HAL is a multi-disciplinary open access archive for the deposit and dissemination of scientific research documents, whether they are published or not. The documents may come from teaching and research institutions in France or abroad, or from public or private research centers.
L'archive ouverte pluridisciplinaire HAL, est destinée au dépôt et à la diffusion de documents scientifiques de niveau recherche, publiés ou non, émanant des établissements d'enseignement et de recherche français ou étrangers, des laboratoires publics ou privés. 


\title{
A combined thermo-mechanical model for Tribological Surface Transformations
}

\author{
G. Antoni ${ }^{\mathrm{a}, \mathrm{c}, *}, \mathrm{~T}$. Désoyer ${ }^{\mathrm{a}, \mathrm{b}}$, F. Lebon ${ }^{\mathrm{a}, \mathrm{c}}$ \\ a Laboratoire de Mécanique et d'Acoustique, UPR CNRS 7051, 31 chemin Joseph-Aiguier, 13402 Marseille Cedex 20, France

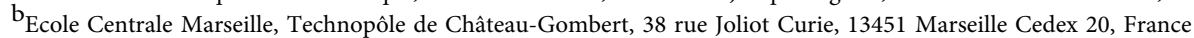 \\ ${ }^{{ }^{c}}$ Université de Provence, 3, place Victor Hugo, 13331 Marseille Cedex 03, France
}

\begin{abstract}
Some of the French railroad network's rails have been affected for the last about twenty years or so by an undesirable physical phenomenon known as Tribological Surface Transformations (TSTs), which are irreversible, quasi-surface solid-solid phase transformations. Although the physical causes of TSTs have not yet been clearly established, it seems likely that the mechanical loads combined with the thermal effects of the wheel/rail contacts may be largely responsible. The present study is based on the assumption that the combined thermo-mechanical constraints may generate solid-solid phase transformations. The thermo-mechanical model developed on these lines was based on previous studies on TRansformation Induced Plasticity (TRIP), which are extended here to account for TSTs. The ability of the model to describe TSTs initiation and development is also assessed by performing a 2-D finite element analysis.
\end{abstract}

Keywords: Tribological Surface Transformations (TSTs), Wheel/rail contact, Thermo-mechanical modelling, Transformation Induced Plasticity (TRIP), Finite element analysis

\section{Introduction}

Tribological Surface Transformations (TSTs) occur in some metallic materials undergoing repeated compression loads. From the metallurgical point of view, these processes correspond to irreversible (permanent) solid-solid phase transformations, the main characteristic of which is that they occur near the surface at which the mechanical loads are applied (Eleöd et al., 1999). Actually, after being initiated, TSTs develop down to a very low depth, which probably depends on the metallic material in question.

The weight and velocity of freight trains have increased over the years, resulting in the emergence of new physical phenomena such as TSTs, which have been observed on some straight parts of the French railroad network. TSTs may be partly caused by heavy corrugation of the rails:

\footnotetext{
* Corresponding author at: Laboratoire de Mécanique et d'Acoustique, UPR CNRS 7051, 31 chemin Joseph-Aiguier, 13402 Marseille Cedex 20, France.

E-mail addresses: antoni@Ima.cnrs-mrs.fr (G. Antoni), desoyer@lma. cnrs-mrs.fr (T. Désoyer), lebon@lma.cnrs-mrs.fr (F. Lebon).
}

the troughs of corrugated rails, like undamaged rails, consist of pearlitic steel, whereas the crests, where TSTs develop, are made of "quasi-martensitic" steel. As shown in Fig. 1, TSTs, which result in what has been called a "White Etching Layer", occur around the surface of the rails (Baumann et al., 1996; Österle et al., 2001).

It has been established that rail corrugation is due to stick-slip processes occurring in the wheel/rail contact area (Sato et al., 2002), but the reason why TSTs develop only on the crests of corrugated rails still remains to be elucidated. The aim of this study was to try to answer this question. The main assumption adopted here was that both the mechanical loads - which are mainly applied to the crests as soon as corrugation is initiated - and the increase in the temperature - due to the friction occurring in the wheel/ rail contact area - are responsible for TSTs, i.e. TSTs are due to a thermo-mechanical coupling process. Accordingly, the thermal stresses occurring in the wheel/rail contact area increase the effects of the mechanical loads, especially at the surface and in the immediate vicinity. This assumption is in line with a comment made by Baumann et al. 


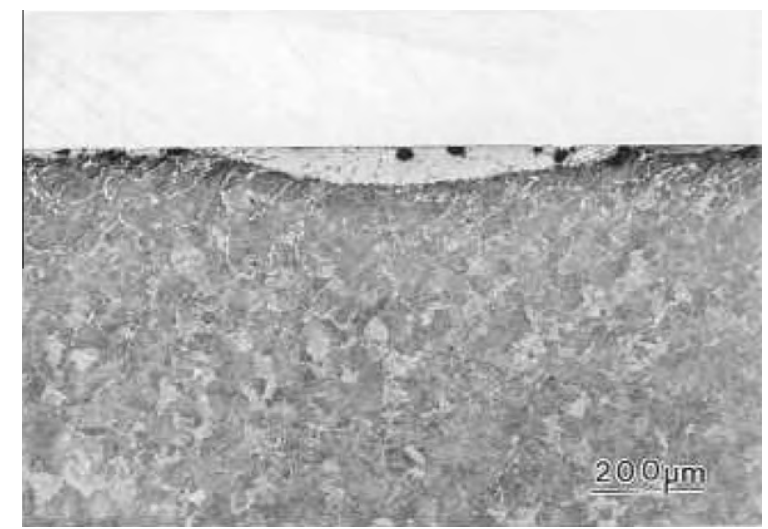

Fig. 1. (extract from Österle et al. (2001)) Transverse cross-section of the upper part of a rail showing TSTs (or "White Etching Layer"). The rail is made of S54/900A steel. The parent phase (the grey part of the picture) is pearlite, which still exists in the main part of the rail when TSTs have developped. The daughter phase (the white part of the picture), occurring at the surface, is "quasi-martensite".

(1996) that “... there is some [experimental] evidence to suggest that the [Tribological Surface] Transformation[s] may be produced by normal and shear stresses superimposed with high thermal stresses limited to a small surface layer."

Calculating the temperature variations due to friction and other heat transfer phenomena between the rail and its environment is a highly involved problem, which is beyond the scope of the present study. Some previous authors have attributed the developpement of TSTs to the presence of high temperatures known as "Flash temperatures" of around $1000{ }^{\circ} \mathrm{C}$ in the wheel/rail contact area during a very short period of only a few seconds (Archard and Rowntree, 1988; Ahlström and Karlsson, 1999). The resulting temperatures are therefore liable to generate solidsolid phase transformations. Flash temperatures have not been measured in practice so far in the case of railway situations problems. On the other hand, Van and Maitournam (1994); Van et al. (1996), have presented numerical results on the temperature field in the rail, based on various hypotheses about the stress field in the wheel/rail contact area. These results tend to show that, in the case of a steadily moving train, the temperature will increase at the surface of the rails by $150{ }^{\circ} \mathrm{C}$ or so at most. This temperature increase alone is obviously too low to generate solid-solid phase transformations; whereas these thermal stresses superimposed on the purely mechanical (isothermal) stresses could result in solid-solid phase transformations.

This paper is organized as follows: a thermodynamically consistent, thermo-mechanical model is first presented in Section 2 (see also Antoni et al. (2009, 2010)). This model is mainly based on previous studies on TRansformation Induced Plasticity (TRIP), such as those by Leblond et al. (1989a,b); Taleb and Sidoroff (2003), which are extended here to include thermo-mechanical coupling in order to account fot TSTs. In Section 3, the numerical integration method used to solve the constitutive equations for the present thermo-mechanical model is briefly presented. This method is based on the Return Mapping
Algorithm (RMA) (see for example Nguyen (1977); Simo and Taylor (1985)). In order to assess the relevance of the main assumption underlying the present study, i.e., that TSTs result from combined thermo-mechanical stresses, a 2-D finite element analysis was performed, which is presented in Section 4.

\section{Irreversible solid-solid phase transformations and TSTs: a thermo-mechanical model}

Permanent solid-solid phase transformations are generally associated in modeling studies with so-called "anomalous" plastic strains: these irreversible strains occur in for states of stress where the Von Mises or Tresca stress is much lower than the initial yield strength of the softest phase. This situation, which is also known as TRansformation Induced Plasticity (TRIP; see for example Greenwood and Johnson (1965)), has been widely studied and, from the purely mechanical point of view, successfully modeled (see, for example, Leblond et al. (1989a,b); Taleb and Sidoroff (2003)). The phenomenological model presented in this Section was based on these previous studies and simply extended to include the main assumption adopted here that TSTs result from a thermo-mechanical coupling process. It takes into account both the "TRIP-like" process and the classical, isochoric plasticity: the latter can also develop, especially under "high" mechanical loadings conditions.

The state variables used here are: the absolute temperature $T>0$; the (total) strain tensor, $\epsilon$, which is decomposed into its spherical and deviatoric parts, $\epsilon=1 / 3$ $\operatorname{Tr}(\boldsymbol{\epsilon}) \boldsymbol{G}+\boldsymbol{e}$ (where $\boldsymbol{G}$ denotes the metric tensor); the mass fraction of the daughter phase, $z \in[0,1]$, where $\rho^{d}$, the partial density of the daughter phase, is given by $\rho^{d}=z \rho$; an isotropic hardening variable associated with classical plasticity, $v$; a TRIP-like strain deviatoric tensor, $\boldsymbol{e}^{p z}$; and a classical plasticity strain deviatoric tensor, $\boldsymbol{e}^{p c}$. The elastic strain deviatoric tensor, $\boldsymbol{e}^{e}$, is defined classically as the difference between the (total) strain deviatoric tensor, the TRIP-like strain deviatoric tensor and the classical plasticity strain deviatoric tensor:

$\boldsymbol{e}^{e}=\boldsymbol{e}-\boldsymbol{e}^{p z}-\boldsymbol{e}^{p c}$

It must be underlined that TRIP-like processes involve a density variation, which is characterized here by $z / \kappa$ and such that:

$\operatorname{Tr}\left(\boldsymbol{\epsilon}^{e}\right)=\operatorname{Tr}(\boldsymbol{\epsilon})-3 \alpha\left(T-T_{i}\right)+\frac{z}{\kappa}$,

where $T_{i}$ denotes the initial temperature, $\alpha>0$ is the coefficient of thermal expansion, and $\kappa>0$ is a material parameter characterizing the density variation during the phase transformation. Note that the phase transformation in question results in a volume decrease, i.e. $-z / \kappa<0$ (martensite density mass, $\rho_{\text {martensite }} \approx 7800 \mathrm{~kg} . \mathrm{m}^{-3}$ and ferrite density mass, $\rho_{\text {ferrite }} \approx 7700 \mathrm{~kg} \cdot \mathrm{m}^{-3}$ ).

Assuming that the material under investigation is initially non-transformed (i.e. $z_{i}=0$ and $\boldsymbol{e}_{i}^{p z}=0$ ), the following expression gives the Helmholtz free energy potential per unit mass: 


$$
\begin{aligned}
\psi\left(T, \operatorname{Tr}(\boldsymbol{\epsilon}), \boldsymbol{e}, \boldsymbol{e}^{p c}, \boldsymbol{e}^{p z}, z, v\right)= & -C_{\boldsymbol{\epsilon}} \frac{\left(T-T_{i}\right)^{2}}{2 T_{i}}+\frac{1}{2 \rho_{i}} h v^{2}+\frac{1}{2 \rho_{i}} \\
& \times \frac{3 \lambda+2 \mu}{3}\left[\operatorname{Tr}(\boldsymbol{\epsilon})+\frac{z}{\kappa}\right]^{2} \\
& +\frac{\mu}{\rho_{i}}\left[\left(\boldsymbol{e}-\boldsymbol{e}^{p c}-\boldsymbol{e}^{p z}\right):\left(\boldsymbol{e}-\boldsymbol{e}^{p c}-\boldsymbol{e}^{p z}\right)\right] \\
& -\frac{1}{\rho_{i}}(3 \lambda+2 \mu) \alpha\left(T-T_{i}\right)\left[\operatorname{Tr}(\boldsymbol{\epsilon})+\frac{z}{\kappa}\right] \\
& +\frac{\delta}{\rho_{i} \kappa}\left[\frac{T_{i}^{z}}{2} z^{2}+\left(T-T_{i}^{z}\right) z\right]+\psi_{0},
\end{aligned}
$$

where $C_{\epsilon}>0$ is the specific heat capacity, $\mu>0$ and $\lambda>-(2 /$ 3) $\mu$ are the Lamé constants, $T_{i}^{z}$ is the solid/solid phase transformation temperature when the pressure is zero, $h>0$ is a material parameter characterizing the linear isotropic hardening (in classical plasticity), and $\delta>0$ is a material parameter characterizing the latent heat associated with the phase transformation. In Eq. (3), $\rho_{i}$ and $\psi_{i}$ are the initial density and the initial Helmholtz free energy of the material per unit mass, respectively. Note that, in a first approximation, the thermo-elastic parameters, $C_{\epsilon}, \mu$, $\lambda$ and $\alpha$ are taken to be identical in the two phases. Eq. (3) was strongly inspired by the model for the 1-D-Helmholtz free energy potential developed by Chrysochoos et al. (2003) for Shape Memory Alloys (SMAs). However, the thermo-mechanical coupling process is extended here to include an irreversible solid-solid phase transformation.

Taking $e$ to denote the internal energy potential per unit mass and writing $e=\psi+T S$, where $S$ is the entropy per unit mass, the local expression for the First Principle of Thermodynamics simply reads (see for example Garrigues, 2007):

$$
\begin{aligned}
\rho \dot{e} & =\boldsymbol{\sigma}: \dot{\boldsymbol{\epsilon}}-\operatorname{div}(\boldsymbol{q}) \Longleftrightarrow \rho T \dot{S}+\operatorname{div}(\boldsymbol{q}) \\
& =\boldsymbol{s}: \dot{\boldsymbol{e}}-\operatorname{PTr}(\dot{\epsilon})-\rho S \dot{T}-\rho \dot{\psi},
\end{aligned}
$$

where $\boldsymbol{q}$ is the heat flux vector and $\boldsymbol{\sigma}$ is the Cauchy stress tensor $(\boldsymbol{\sigma}=-P \boldsymbol{G}+\boldsymbol{s}$, where the pressure $P$ is such that $P=-1 / 3 \operatorname{Tr}(\sigma))$; for the sake of simplicity, remote heat production rate has been omitted.

The Second Principle of Thermodynamics must be satisfied (see Garrigues, 2007): i - whatever the local thermomecanical state of the material may be, $(T, \operatorname{Tr}(\boldsymbol{\epsilon}), z, v, \boldsymbol{e}$, $\left.\boldsymbol{e}^{p z}, \boldsymbol{e}^{p c}\right)$; ii - whatever the evolution locally undergone by the material, $(\dot{T}, \operatorname{Tr}(\dot{\boldsymbol{\epsilon}}), \dot{\boldsymbol{e}})$; iii - whatever the temperature gradient locally acting on the material, $\nabla T$, i.e.:

$$
\begin{aligned}
-\frac{1}{T} \boldsymbol{q} \cdot \nabla T+\rho T \dot{S}+\operatorname{div}(\boldsymbol{q}) \geqslant 0 \quad & \forall\left(T, \operatorname{Tr}(\boldsymbol{\epsilon}), z, v, \boldsymbol{e}, \boldsymbol{e}^{p z}, \boldsymbol{e}^{p c}\right), \\
& \forall(\dot{T}, \operatorname{Tr}(\dot{\boldsymbol{\epsilon}}), \dot{\boldsymbol{e}}), \forall \nabla T
\end{aligned}
$$

By combining Eqs. (4) and (5), the Clausius-Duhem inequality can be immediately obtained, which shows that the dissipated (per unit volume) power, $\phi$, is always non negative:

$$
\begin{aligned}
\phi= & -\frac{1}{T} \boldsymbol{q} \cdot \nabla T-\rho\left(S+\frac{\partial \psi}{\partial T}\right) \dot{T}+\left(\boldsymbol{s}-\rho \frac{\partial \psi}{\partial \boldsymbol{e}}\right): \dot{\boldsymbol{e}} \\
& -\left(P-\rho \frac{\partial \psi}{\partial \operatorname{Tr}(\boldsymbol{\epsilon})}\right): \operatorname{Tr}(\dot{\boldsymbol{\epsilon}})-\rho \frac{\partial \psi}{\partial \boldsymbol{e}^{p z}}: \dot{\boldsymbol{e}}^{p z}-\rho \frac{\partial \psi}{\partial z} \dot{z} \\
& -\rho \frac{\partial \psi}{\partial \boldsymbol{e}^{p c}}: \dot{\boldsymbol{e}}^{p c}-\rho \frac{\partial \psi}{\partial v} \dot{v} \geqslant 0 \\
\forall(T, & \left.\operatorname{Tr}(\boldsymbol{\epsilon}), z, v, \boldsymbol{e}, \boldsymbol{e}^{p z}, \boldsymbol{e}^{p c}\right), \forall(\dot{T}, \operatorname{Tr}(\dot{\boldsymbol{\epsilon}}), \dot{\boldsymbol{e}}), \forall \nabla T
\end{aligned}
$$

Assuming that neither $\dot{z}$, nor $\dot{\boldsymbol{e}}^{p z}$ depend on $\dot{T}$, and due to the fact that the specific entropy $S$ is a state function, a first necessary and sufficient condition for the Clausius-Duhem inequality to be systematically satisfied is:

$S=-\frac{\partial \psi}{\partial T}$

Neglecting the visco-elastic effects (or, alternatively, assuming the Cauchy stress tensor to be a state function), a second necessary and sufficient condition for the Clausius-Duhem inequality to be systematically satisfied is:

$\boldsymbol{s}=\rho \frac{\partial \psi}{\partial \boldsymbol{e}} \quad$ and $\quad P=-\rho \frac{\partial \psi}{\partial \operatorname{Tr}(\boldsymbol{\epsilon})}$

Based on Eqs. (7) and (8), Eq. (6) reduces to:

$$
\begin{aligned}
& -\frac{1}{T} \boldsymbol{q} . \nabla T-\rho \frac{\partial \psi}{\partial \boldsymbol{e}^{p z}}: \dot{\boldsymbol{e}}^{p z}-\rho \frac{\partial \psi}{\partial z} \dot{z}-\rho \frac{\partial \psi}{\partial \boldsymbol{e}^{p c}}: \dot{\boldsymbol{e}}^{p c}-\rho \frac{\partial \psi}{\partial v} \dot{v} \geqslant 0 \\
& \forall\left(T, \operatorname{Tr}(\boldsymbol{\epsilon}), z, v, \boldsymbol{e}, \boldsymbol{e}^{p z}, \boldsymbol{e}^{p c}\right), \forall(\dot{T}, \operatorname{Tr}(\dot{\boldsymbol{\epsilon}}), \dot{\boldsymbol{e}}), \forall \nabla T
\end{aligned}
$$

or, alternatively, based on Eqs. (3) and (8), from which $\rho\left(\partial \psi / \partial \boldsymbol{e}^{p z}\right)=-\rho(\partial \psi / \partial \boldsymbol{e})=-\boldsymbol{s}:$

$$
\begin{aligned}
& -\frac{1}{T} \boldsymbol{q} \cdot \nabla T-\rho \frac{\partial \psi}{\partial z} \dot{z}+\boldsymbol{s}: \dot{\boldsymbol{e}}^{p z}-\rho \frac{\partial \psi}{\partial v} \dot{v}+\boldsymbol{s}: \dot{\boldsymbol{e}}^{p c} \geqslant 0 \\
& \forall\left(T, \operatorname{Tr}(\boldsymbol{\epsilon}), z, v, \boldsymbol{e}, \boldsymbol{e}^{p z}, \boldsymbol{e}^{p c}\right), \forall(\dot{T}, \operatorname{Tr}(\dot{\boldsymbol{\epsilon}}), \dot{\boldsymbol{e}}), \forall \nabla T
\end{aligned}
$$

Fourier's law, $\boldsymbol{q}=-k \nabla T$, where $k>0$ denotes the thermal conductivity, therefore turns out to be a sufficient condition for the previous inequality to be systematically satisfied. Assuming that neither $\dot{z}$, nor $\dot{\boldsymbol{\epsilon}}^{p z}$ depend on $\nabla T$, another sufficient condition for the inequality Eq. (10) to be systematically satisfied is:

$$
\begin{aligned}
& \boldsymbol{s}: \dot{\boldsymbol{e}}^{p z}-\rho \frac{\partial \psi}{\partial z} \dot{z}+\boldsymbol{s}: \dot{\boldsymbol{e}}^{p c}-\rho \frac{\partial \psi}{\partial v} \dot{v} \geqslant 0 \\
& \forall\left(T, \operatorname{Tr}(\boldsymbol{\epsilon}), z, v, \boldsymbol{e}, \boldsymbol{e}^{p z}, \boldsymbol{e}^{p c}\right), \forall(\dot{T}, \operatorname{Tr}(\dot{\boldsymbol{\epsilon}}), \dot{\boldsymbol{e}}), \forall \nabla T
\end{aligned}
$$

The equations of evolution of the internal state variables associated with the TRIP-like process $\left(z\right.$ and $\left.\boldsymbol{e}^{p z}\right)$ and the classical isochoric plasticity process ( $v$ and $\boldsymbol{e}^{p c}$ ) are then classically defined by: $\mathrm{i}$ - a yield function; ii - a non negative, viscoplastic multiplier; iii - a flow rule, namely:

- The TRIP-like process:

- Yield function:

$$
f^{p z}(T, P)=\frac{T}{T_{i}^{z}}-\exp \left(-\frac{\langle P\rangle}{\omega}\right)
$$

where $P=-1 / 3 \operatorname{Tr}(\sigma)$ is the pressure, $\omega>0$ is a material parameter characterizing a "pressure sensitivity" level, and $\langle\cdot\rangle$ denotes the Macaulay brackets $(\langle x\rangle=x$ when $x \geqslant 0$ and $\langle x\rangle=0$ when $x<0$ ).

- Viscoplastic multiplier $\dot{p}$ and flow rule:

$$
\begin{aligned}
& \dot{\boldsymbol{e}}^{p z}=\dot{p} \frac{3}{2 \sigma_{e q}} \boldsymbol{s} ; \quad \dot{z}=\kappa \dot{p} \\
& \text { with } \quad \dot{p}=\frac{\langle 1-z\rangle}{\eta}\left\langle f^{p z}(T, P)\right\rangle H\left(P+\delta\left[(1-z) T_{i}^{z}-T\right]\right) \geqslant 0
\end{aligned}
$$

where $\eta$ denotes the characteristic time of the viscous effects associated with the TRIP-like process and $H(\cdot)$ denotes the Heaviside step function $(H(x)=1$ when $x \geqslant 0$ and $H(x)=0$ when $x<0$ ).

- The classical isochoric plasticity process:

- Yield function: 


$$
f^{p c}(\boldsymbol{\sigma}, v)=\sigma_{e q}-\left(\sigma_{y}+h v\right) \quad \text { with } \quad \sigma_{e q}=\left(\frac{3}{2} \boldsymbol{s}: \boldsymbol{s}\right)^{\frac{1}{2}}
$$

where $\sigma_{e q}$ is the Von Mises equivalent stress, $\boldsymbol{s}$ is the stress deviator and $\sigma_{y}$ is the classical yield strength.

- Viscoplastic multiplier $v$ and flow rule:

$$
\dot{\boldsymbol{e}}^{p c}=\dot{v} \frac{\partial f^{p c}}{\partial \boldsymbol{\sigma}}=\dot{v} \frac{3}{2 \sigma_{e q}} \boldsymbol{s} \quad \dot{v}=\frac{\left\langle f^{p c}(\boldsymbol{\sigma}, v)\right\rangle}{\xi \sigma^{y}} \geqslant 0
$$

where $\xi$ denotes the characteristic time of the viscous effects associated with the classical plasticity.

It should be stressed that Eqs. (12)-(15) are thermodynamically consistent, i.e. they systematically satisfy the inequality described by Eq. (11). It should also be noted that: i - Eq. (12) reflects the fact that the solid/solid phase transformation is activated by the temperature, which acts as a "driving force", and accelerated by the pressure. This "pressure sensitivity" is in agreement with Baumann et al. (1996) who have established that applying of a hydrostatic pressure decreases the temperature of the $\alpha \rightarrow \gamma$ phase transformation; ii - Eq. (13)-c means that the visco-plastic multiplier will always be zero whenever $z$ reaches its maximum value $(z=1)$, as well as when a pressure state and temperature condition has been met, i.e. $P+\delta\left[(1-z) T_{i}^{z}-T\right] \leqslant 0$; iii - Eq. (13)-b means that $\dot{z}$ cannot be negative, which is consistent with the fact that the solidsolid phase transformations involved in the present study are irreversible; iv - when the characteristic times of the viscous effects, associated with the TRIP-like plasticity ( $\eta$, see Eq. (13)-c) and the classical plasticity ( $\xi$, see Eq. (15)b), tend to zero, a rate-independent model is obtained.

Lastly, based on Eqs. (3) and (8), and assuming that $\rho \approx \rho_{i}$ whatever the local thermo-mechanical state of the material may be, the Cauchy stress tensor $\boldsymbol{\sigma}$ reads:

$\boldsymbol{\sigma}=-\frac{(3 \lambda+2 \mu)}{3}\left[\operatorname{Tr}(\boldsymbol{\epsilon})+\frac{z}{\kappa}-3 \alpha\left(T-T_{i}\right)\right] \boldsymbol{G}+2 \mu\left(\boldsymbol{e}-\boldsymbol{e}^{p z}-\boldsymbol{e}^{p c}\right)$

Upon combining Eqs. (3), (4), (7) and (8) and Fourier's law, the heat equation is given by:

$$
\begin{aligned}
\rho_{i} C_{\epsilon} \frac{T}{T_{i}} \dot{T}-k \Delta T= & -(3 \lambda+2 \mu) \alpha \operatorname{Tr}(\dot{\boldsymbol{\epsilon}})+\boldsymbol{s}:\left(\dot{\boldsymbol{e}}^{p c}+\dot{\boldsymbol{e}}^{p z}\right)-h v \dot{v} \\
& +\frac{1}{\kappa}\left[P-(3 \lambda+2 \mu) \alpha T+\delta(1-z) T_{i}^{z}\right] \dot{z}
\end{aligned}
$$

where " $\Delta$ " denotes the Laplacian. The term on the righthand side of Eq. (17) corresponds to the thermo-elasticity, the TRIP-like plasticity and the classical plasticity and the latent heat associated with the solid-solid phase transformation.

\section{Numerical implementation}

The constitutive equations involved in the present model (see Section 2) were implemented numerically using a Return Mapping Algorithm (RMA, see for example Nguyen, 1977; Simo and Taylor, 1985).

Assuming the mechanical state to be known at time $t_{n}, \mathcal{S}_{n}=\left(\operatorname{Tr}\left(\boldsymbol{\epsilon}_{n}\right), z_{n}, v_{n}, \boldsymbol{e}_{n}, \boldsymbol{e}_{n}^{p z}, \boldsymbol{e}_{n}^{p c} ; \boldsymbol{\sigma}_{n}\right)$, with a given strain increment $\Delta \epsilon_{n}^{(k)}=\epsilon_{n+1}^{(k)}-\epsilon_{n}$ (see Comment 1), the Cauchy stress tensor at time $t_{n+1}, \boldsymbol{\sigma}_{n+1}^{(k)}$, can be expressed as follows:

$$
\boldsymbol{\sigma}_{n+1}^{(k)}=\mathcal{F}\left(\mathcal{S}_{n}, \Delta \boldsymbol{\epsilon}_{n}^{(k)}\right)
$$

where $\mathcal{F}$ summarizes the effects of the Return Mapping Algorithm (see Comment 2).

Comment 1: A classical Newton-Raphson algorithm is used to solve the overall, equilibrium problem. The variables denoted by $(\cdot)_{n}^{(k)}$ correspond to the $\mathrm{k}^{\text {th }}$ equilibrium iteration at time $t_{n}$.

Comment 2: Based on Eq. (17), a strong coupling can be taken a priori between the temperature evolution, $\dot{T}$, and the mechanical evolution. Preliminary calculations have clearly shown, however, that the solution of Eq. (17) - in the case of a similar problem to that considered in Section 4 - is very similar to that of the "classical" heat equation, i.e. Eq. (17) with zero on the righthand side. The strong thermo-mechanical coupling is therefore not taken into account in the numerical implementation: throughout this Section, the temperature state, $T_{n}$, and the increment, $\Delta T_{n}=T_{n+1}-T_{n}$, are assumed to be known.

Comment 3: For the sake of numerical simplicity, and in line with Comment 2, the material parameter $\delta$ is taken to be zero.

The main steps in the RMA algorithm applied to the present thermo-mechanical model can be summarized as follows (where the time step is simply denoted by $\left.\Delta t_{n}=t_{n+1}-t_{n}\right):$

(i) Based on Eq. (16), the "trial", elastic stress deviator, $\boldsymbol{s}_{n+1}^{e l,(k)}$, and the associated "trial", thermo-elastic stress tensor, $\boldsymbol{\sigma}_{n+1}^{e l,(k)}$, are calculated as follows:

$$
\begin{aligned}
& \boldsymbol{s}_{n+1}^{e l,(k)}=\boldsymbol{s}_{n}+2 \mu \Delta \boldsymbol{e}_{n}^{(k)} ; \boldsymbol{\sigma}_{n+1}^{e l,(k)}=-P_{n+1}^{e l,(k)} \boldsymbol{G}+\boldsymbol{s}_{n+1}^{e l,(k)} \\
& \text { with } P_{n+1}^{e l,(k)}=-\left[\frac{1}{3} \operatorname{Tr}\left(\boldsymbol{\sigma}_{n}\right)+K \operatorname{Tr}\left(\Delta \boldsymbol{\epsilon}_{n}^{(k)}\right)-3 K \alpha \Delta T_{n}\right]
\end{aligned}
$$

where $\boldsymbol{s}_{n}=\boldsymbol{\sigma}_{n}-\frac{1}{3} \operatorname{Tr}\left(\boldsymbol{\sigma}_{n}\right) \boldsymbol{G}, \boldsymbol{e}_{n}=\boldsymbol{\epsilon}_{n}-\frac{1}{3} \operatorname{Tr}\left(\boldsymbol{\epsilon}_{n}\right) \boldsymbol{G}$ and $K=(3 \lambda+$ $2 \mu) / 3$ (the bulk modulus).

(ii) Based on Eqs. (12) and (14), the 2 yield criteria are tested (four cases):

(a) if $f^{p z}\left(T_{n+1}, P_{n+1}^{e l,(k)}\right) \leqslant 0$ and $f^{p c}\left(\boldsymbol{\sigma}_{n+1}^{e l,(k)},-h v_{n}^{(k)}\right) \leqslant 0$, then go to (iv)-(a),

(b) if $f^{p z}\left(T_{n+1}, P_{n+1}^{e l,(k)}\right)>0$ and $f^{p c}\left(\boldsymbol{\sigma}_{n+1}^{e l,(k)},-h v_{n}^{(k)}\right) \leqslant 0$, then go to (iii)-(a),

(c) if $f^{p z}\left(T_{n+1}, P_{n+1}^{e l,(k)}\right)>0$ and $f^{p c}\left(\boldsymbol{\sigma}_{n+1}^{e l,(k)},-h v_{n}^{(k)}\right)>0$, then go to (iii)-(b),

(d) if $f^{p z}\left(T_{n+1}, P_{n+1}^{e l,(k)}\right) \leqslant 0$ and $f^{p c}\left(\boldsymbol{\sigma}_{n+1}^{e l,(k)},-h v_{n}^{(k)}\right)>0$, then go to (iii)-(c),

(iii) Based on Eqs. (13) and (15), $\Delta p_{n}^{(k)} \geqslant 0$ (see Comment 4) and $\Delta v_{n}^{(k)} \geqslant 0$ are computed as follows:

(a) in the case of a thermo-elasto-viscoplastic iteration (corresponding only to the TRIP-like process)

$\Delta p_{n}^{(k)}=\Delta t_{n} \frac{\left\langle 1-z_{n}\right\rangle}{\eta}\left(\frac{T_{n+1}}{T_{i}^{z}}-\exp \left(-\frac{\left\langle P_{n}\right\rangle}{\omega}\right)\right) H\left(P_{n}\right) ;$

$\Delta v_{n}^{(k)}=0$

(b) in the case of a thermo-elasto-viscoplastic iteration (corresponding to the TRIP-like process and classical plasticity): 


$$
\left\{\begin{array}{l}
\Delta p_{n}^{(k)}=\Delta t_{n} \frac{\left\langle 1-z_{n}\right\rangle}{\eta}\left(\frac{T_{n+1}}{T_{i}^{2}}-\exp \left(-\frac{\left\langle P_{n}\right\rangle}{\omega}\right)\right) H\left(P_{n}\right) \\
\Delta v_{n}^{(k)}=\frac{\left(\sigma_{n+1}^{e l,(k)}\right)_{e q}-3 \mu \Delta p_{n}^{(k)}-\left(\sigma_{y}+h v_{n}\right)}{\frac{\sigma y \xi}{\Delta t_{n}}+3 \mu+h}
\end{array}\right.
$$

(c) in the case of a thermo-elasto-viscoplastic iteration (corresponding only to the classical plasticity):

$$
\Delta v_{n}^{(k)}=\frac{\left(\sigma_{n+1}^{e l,(k)}\right)_{e q}-\left(\sigma_{y}+h v_{n}\right)}{\frac{\sigma_{y} \xi}{\Delta t_{n}}+3 \mu+h} ; \quad \Delta p_{n}^{(k)}=0
$$

(iv) From Eqs. (12)-(15), updating the mechanical state (two cases) gives:

(a) in the case of a thermo-elastic iteration [(ii)-(a)]:

$$
\begin{aligned}
& \boldsymbol{\sigma}_{n+1}^{(k)}=\boldsymbol{\sigma}_{n+1}^{e l,(k)} ; \quad \boldsymbol{\epsilon}_{n+1}^{p z,(k)}=\boldsymbol{\epsilon}_{n}^{p z} ; \quad \boldsymbol{\epsilon}_{n+1}^{p c,(k)}=\boldsymbol{\epsilon}_{n}^{p c} ; \\
& z_{n+1}^{(k)}=z_{n} ; \quad v_{n+1}^{(k)}=v_{n}
\end{aligned}
$$

(b) in the case of a thermo-elasto-viscoplastic iteration [(ii)-(b), (ii)-(c), (ii)-(d)]:

$$
\begin{aligned}
\boldsymbol{\sigma}_{n+1}^{(k)}= & -\left(P_{n+1}^{e l,(k)}-K \Delta p_{n}^{(k)}\right) \boldsymbol{G}+\boldsymbol{s}_{n+1}^{e l,(k)} \\
& -2 \mu\left[\Delta \boldsymbol{\epsilon}_{n}^{p z,(k)}+\Delta \boldsymbol{\epsilon}_{n}^{p c,(k)}\right] ; \\
\boldsymbol{\epsilon}_{n+1}^{p z,(k)}= & \boldsymbol{\epsilon}_{n}^{p z}+\Delta \boldsymbol{\epsilon}_{n}^{p z,(k)} ; \boldsymbol{\epsilon}_{n+1}^{p c,(k)}=\boldsymbol{\epsilon}_{n}^{p c}+\Delta \boldsymbol{\epsilon}_{n}^{p z,(k)} ; \\
z_{n+1}^{(k)}= & z_{n}+\kappa \Delta p_{n}^{(k)} ; \quad v_{n+1}^{(k)}=v_{n}+\kappa \Delta v_{n}^{(k)}
\end{aligned}
$$

where the TRIP-like strain increment, $\Delta \epsilon_{n}^{p z,(k)}$, and the (classical) plastic strain increment, $\Delta \boldsymbol{\epsilon}_{n}^{p c,(k)}$, are given by:

$\Delta \boldsymbol{\epsilon}_{n}^{p z,(k)}=\frac{3 \Delta p_{n}^{(k)}}{2\left(\sigma_{n+1}^{e l,(k)}\right)_{e q}} \boldsymbol{s}_{n+1}^{e l,(k)} ; \quad \Delta \boldsymbol{\epsilon}_{n}^{p c,(k)}=\frac{3 \Delta v_{n}^{(k)}}{2\left(\sigma_{n+1}^{e l,(k)}\right)_{e q}} \boldsymbol{s}_{n+1}^{e l,(k)}$

based on Eqs. (13)-1 and (15)-1.

Comment 4: A semi-explicit algorithm is used for the RMA. In fact, the implicit approach is used for the RMA except for the calculation of the yield criterion (explicit type).

Comment 5: In order to accelerate the RMA convergence, the consistent tangent operator (fourth order tensor) was used along with the Newton-Raphson algorithm to solve the overall equilibrium problem.

Using the numerical procedure outlined in this Section, the present model was implemented in a finite element software program (Code_Aster; EdF).

\section{Illustrative examples}

Preliminary remark: The case of the structure studied in this section is not representative of the complex processes occurring in part of a rail when a train is crossing over it (which we have called the wheel/rail problem here). It was simply intended to show that it is possible by combining the behavioral model presented in Section 2 with a temperature field and specific mechanical boundary conditions to model the main characteristic of TSTs, namely the fact that they occur almost only at the surface. More specifically, we addressed this problem in two steps, as follows:
Step 1 (Section 4.1): solving the purely thermal problem with purely temperature related boundary conditions in the stationary case. The non-uniform temperature field obtained in this way is used as a datum in the second step.

Step 2 (Section 4.2): solving the mechanical problem with non-uniform pressure-related boundary conditions imposed on part of the boundary and increasing linearly with time. In the iterative procedures used to solve this mechanical problem (see Section 3), the temperature field calculated in the first step serves as a parameter. It is used in particular when defining the initial conditions of the mechanical problem, via the spherical deformation field which can be taken to occur if one assumes the initial stress field to be zero, and in the tests performed at each iteration on the yield function of the solid/solid phase transformation (see Eq. (12)).

Let $\Omega$ be a square, material domain (measuring $100 \mathrm{~mm}$ on each side) in the $x-y$ plane. The boundary of $\Omega$ is denoted by $\partial \boldsymbol{\Omega}$. For the sake of convenience, $\partial \boldsymbol{\Omega}$ will subsequently be divided into six line segments, namely (see also Fig. 2):

$\partial \Omega=[A, B] \cup[B, C] \cup[C, D] \cup[D, E] \cup[E, F] \cup[F, A]$

where $[B, C]$ is $10 \mathrm{~mm}$ in length. A finite element mesh is generated in $\Omega$, which is composed of approximately 20 000 quadratic elements. Since the most physically relevant results will be located in the central, upper part of $\Omega$, the mesh is refined in the vicinity of the line segment $[B, C]$ (see Fig. 2). Note that this mesh is systematically used in all the numerical simulations presented in this Section.

\subsection{Thermal (preliminary) problem}

As mentioned in Section 3 (see Comment 2), the strong thermo-mechanical coupling, inherent to the present thermo-mechanical model, is not taken into account in the numerical implementation, where the temperature field is used as an initial datum (in the case of "weak" thermo-mechanical coupling). Due to some thermal boundary conditions, the temperature field acting in $\Omega$ can therefore be computed without any need for mechanical considerations.

This temperature field is plotted in Fig. 3, in the steady case, i.e., with $\Delta T=0$. The temperature field is assumed to be a plane field and the thermal, boundary conditions considered are taken to be such that the mechanical boundary conditions do not need to be taken into account in this purely thermal problem:

$$
\begin{aligned}
& T=T_{i}=300 K \quad \text { on } \quad[A, B] \cup[C, D] \cup[D, E] \cup[E, F] \cup[F, A] \\
& T=T^{d}=450 K \quad \text { on } \quad[B, C]
\end{aligned}
$$

Note that, assuming the room temperature to be equal to the initial temperature, $300 \mathrm{~K}$, the greatest increase in the temperature is $150 \mathrm{~K}$, on $[B, C]$, which is in agreement with the results obtained by Van and Maitournam (1994). 


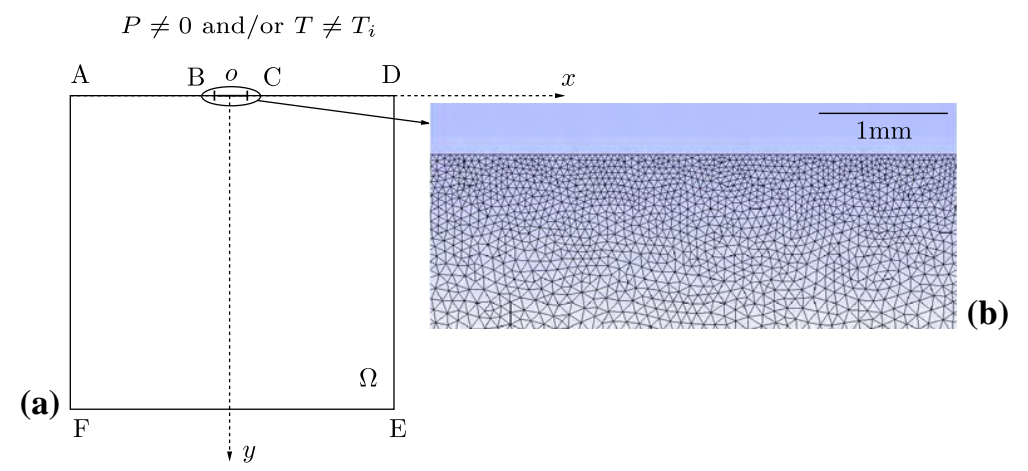

Fig. 2. (a) Square, material domain (measuring $100 \mathrm{~mm}$ on each side) in the $x-y$ plane (origin: $o$ ) and boundary conditions on $[B, C]$ (where $P$ denotes the pressure); (b) Zoom of the refined mesh.

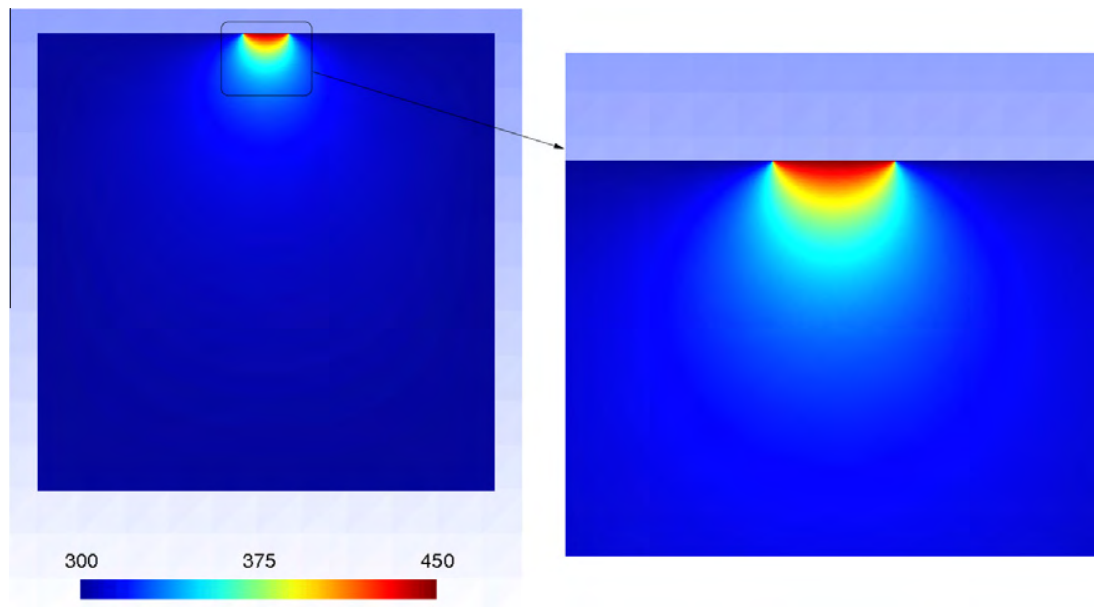

Fig. 3. Steady temperature field (thermal problem); temperature unit: K.

It is worth noting that the stationary thermal problem addressed in this section is quite different from the wheel/rail problem.

\section{2. (Thermo-)mechanical problem}

As previously mentioned, the temperature field presented in Section 4.1 is used as an initial datum for dealing with the (thermo-)mechanical problem. For the local equations of mechanical equilibrium to be satisfied, the stress field at the same time, $\sigma_{0}$, must be zero. Based on Eq. (16) - and due to the fact that neither the TRIP-like process, nor the classical plasticity are triggered by the purely thermal loading -, the following condition must then be satisfied locally:

$\sigma_{0}=0 \Longleftrightarrow \operatorname{Tr}\left(\epsilon_{0}\right)=3 \alpha\left(T_{0}-T_{i}\right)$

where $\alpha$ is the thermal expansion coefficient (the value used to compute this coefficient is $12 \times 10^{-6} \mathrm{~K}^{-1}$ ).

Eq. (28) means that a non-zero, spherical strain field exists at the initial time, which is another initial data. A plane strain state is assumed. The mechanical boundary conditions are as follows:

$$
\begin{gathered}
\boldsymbol{u}=0 \text { on }[E, F] ; \boldsymbol{\sigma} \cdot \boldsymbol{n}=0 \text { on }[A, B] \cup[C, D] \cup[D, E] \cup[F, A] ; \\
\boldsymbol{\sigma} \cdot \boldsymbol{n}=-\dot{P}^{d} t\left(1-\left(\frac{x}{5}\right)^{2}\right)^{1 / 2} \boldsymbol{n} \text { on }[B, C] \\
\text { with } \dot{P}^{d}=100 \mathrm{MPa}^{-1}
\end{gathered}
$$

It can be seen from Eq. (29)-c that the pressure on [B, C] is maximum at $\boldsymbol{x}=0$ and equal to zero at $\boldsymbol{x}=\mp 5 \mathrm{~mm}$ (at points $B$ and $C$ ). The mechanical parameters of the material are as follows:

$\lambda=115 \times 10^{3} \mathrm{MPa} ; \mu=77 \times 10^{3} \mathrm{MPa} ; \rho_{i}=78 \times 10^{-7} \mathrm{~kg} \cdot \mathrm{mm}^{-3}$; $T_{i}^{z}=1000 \mathrm{~K} ; \omega=700 \mathrm{MPa} ; \kappa=10^{2} ; \eta=10^{3} \mathrm{~s} ; \delta=0$;

$\sigma_{y}=400 \mathrm{MPa} ; h=2 \times 10^{4} \mathrm{MPa} ; \xi=2.5 .10^{2} \mathrm{~s}$

The Young's modulus $E$ and the Poisson's ratio $v$ are immediatly deduced from the Lamé parameters $\lambda$ and $\mu$, i.e. $E=210 \mathrm{GPa}$ and $v=0.25$. Note also that the characteristic times of the viscous effects associated with the TRIP-like process, $\eta$, and the classical plasticity, $\xi$, are small enough - with respect to the characteristic loading time, i.e. $t^{l} \approx E / \dot{P}^{d}=2100 \mathrm{~s}-$ for the viscous effects to be actually negligible. 


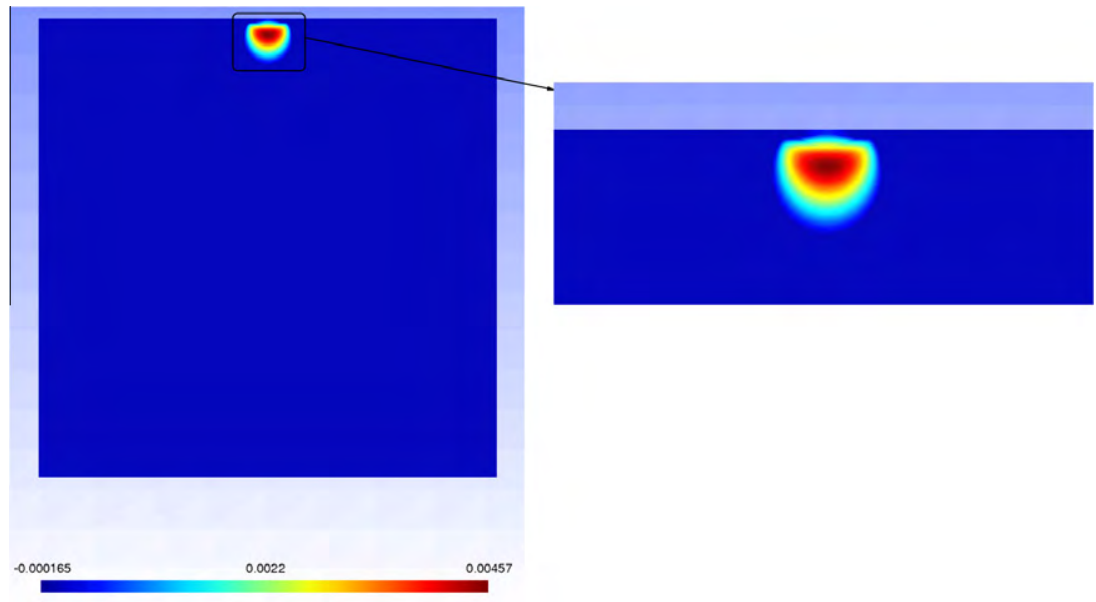

Fig. 4. (Classical plasticity) $v$-field at time $t=10 \mathrm{~s}$.

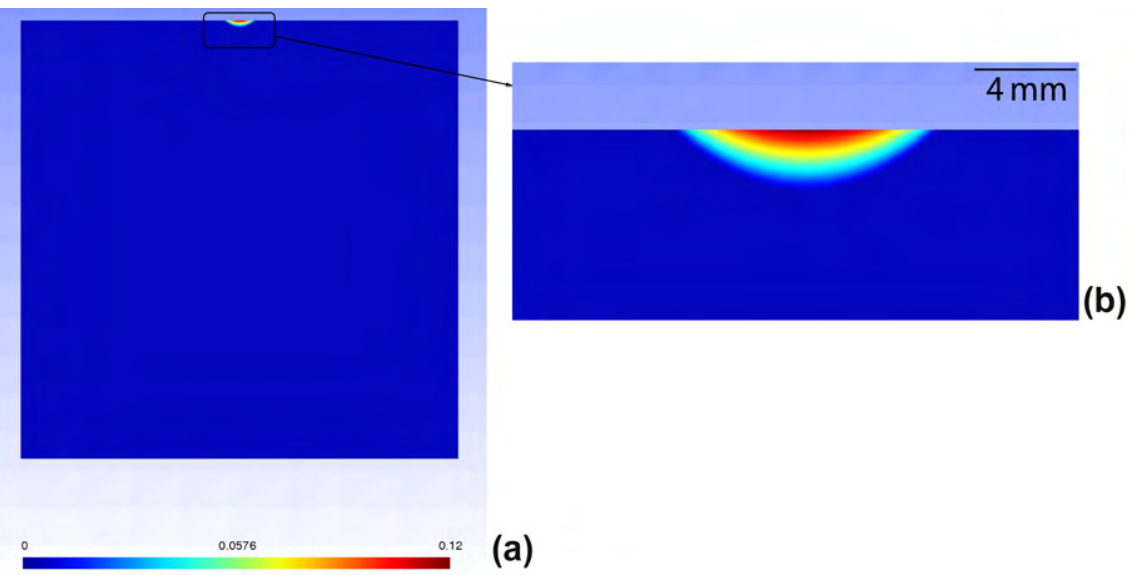

Fig. 5. (TRIP-like process)(a) $z$-field at time $t=10 \mathrm{~s}$; (b) Zoom of the $z$-field.

The $v$-field at time $t=10 \mathrm{~s}$ is plotted in Fig. 4 . Note that $v$ is zero on $[B, C]$, where the mechanical loading is applied, and reaches a maximum value around $45.10^{-4}$ in $\Omega$. Note also that there exists a small area, just below the surface, where both $v$ and $z$ (see Fig. 5) are non zero: the TRIP-like process and classical plasticity may develop simultaneously and have a reciprocal effect.

The $z$-field is plotted in Fig. 5 at time $t=10 \mathrm{~s}$, when $P(x=0)=1000 \mathrm{MPa}$. Note that, from the qualitative point of view, the $z$-field obtained here shows similarities to that observed experimentally in the TSTs (see Fig. 1). In addition, the maximum phase transformation occurs in the immediate vicinity of the surface, where the mechanical loading is applied. But, from the quantitative point of view, there are two main differences with the experimental TSTs data: i the global layer transformed is deeper; ii - the layer transformed near the surface is only partially transformed. Actually, the $z$-field observed is highly non-uniform: for example, along the $0-y$ axis (see Fig. 2 ), $z=z_{\max } \approx 12 \times 10^{-2}$ when $y=0$ - the partial transformation at the point where the mechanical loading is applied - and $z=6 \times 10^{-2}$ when $y \approx 9 \times 10^{-1} \mathrm{~mm}$. The maximum depth transformed is about $17 \times 10^{-1} \mathrm{~mm}$ : beyond this depth, the transformation is zero. These results show that the area transformed is restricted to just a few millimeters below the surface $[B, C]$, but this finding is not exactly in agreement with the experimental data, especially those relating to TSTs on railway tracks, which tend to show that the $z$-field is quasi-uniform $(z=1$; see Fig. 1$)$. One possible means of partly solving this problem might consist in changing the $z$-evolution equation as follows:

$$
\begin{aligned}
& \dot{z}=\kappa \dot{p} \\
& \text { with } \dot{p}=\frac{H(1-z)}{\eta}\left\langle f^{p z}(T, P)\right\rangle H\left(P+\delta\left[(1-z) T_{i}^{z}-T\right]\right)
\end{aligned}
$$

The visco-plastic multiplier given by Eq. (31) is greater than that given by Eq. (13), where the Macaulay brackets are used instead of the Heaviside step function. Consequently, $z$ should therefore be greater in the transformed layer; and a clear-cut boundary can therefore be obtained 
between the transformed zone and the non-transformed zone.

Note also that the tangential stress may also contribute to the emergence of TSTs. The yield criterion associated with the TRIP-like process (see Eq. (12)) can be therefore adapted as follows in order to take the tangential stress sensitivity into account:

$f^{p z}(T, P)=\frac{T}{T_{i}^{z}}-\exp \left(-\frac{\langle P\rangle}{\omega}\right) \exp \left(-\frac{\sigma_{e q}}{\omega}\right)$

where $\sigma_{e q}$ is the Von Mises, equivalent stress. The effects of this change in the present thermo-mechanical model in this paper will have to be studied more closely.

\section{Conclusion}

Based on the assumption that TSTs are due to combined thermo-mechanical loads, a thermodynamically consistent thermo-mechanical model was developed. Apart from the classical plasticity parameters $\left(\sigma_{y}, h\right.$ and $\left.\xi\right)$, five new material parameters $\left(T_{i}^{z}, \omega, \delta, \kappa\right.$ and $\left.\eta\right)$ are introduced into the model: these parameters will have to be identified experimentally in future studies. A classical Return Mapping Algorithm was used for the numerical implementation of the model, using a finite element software program. In order to confirm the main assumption adopted in this study - that TSTs are initiated and develop in the immediate vicinity of the points at which the thermo-mechanical loading is applied -, some realistic numerical simulations were performed. The results obtained were satisfactory but suggest that: $\mathrm{i}$ - the $z$-evolution equation needs to be improved to obtain a more realistic transformed zone; ii - the tangential stress may contribute decisively to the occurrence and the development of TSTs. The relevance of the behavioral model for the TSTs occurring in the railway context can only be tested by taking the contact/friction between wheel and rail into account. These nonstationary interactions are highly complex, mainly because the surfaces in contact are rough surfaces. Some data on this topic are available in the literature, mainly in the form of general formulae (see in particular Liu and Wang (2003)).

\section{References}

Ahlström, J., Karlsson, B., 1999. Microstructural evaluation and interpretation of the mechanically ant thermically affected zone under railway wheel flats. Wear 232, 1-14.

Antoni, G., Désoyer, T., Lebon, F., 2009. A thermo-mechanical modelling of the Tribological Transformations of Surface. Comptes Rendus Mécanique 337, 653-658.

Antoni, G., Désoyer, T., Lebon, F., 2010, Tribological Transformations of Surface: a thermo-mechanical modelling. The Tenth International Conference on Computational Structures Technology, 14p. (CD-ROM).

Archard, J.F., Rowntree, R.A., 1988. The temperature of rubbing bodies; the distribution of temperatures. Wear 128, 1-17.

Baumann, G., Fecht, H.J., Liebelt, S., 1996. Formation of white-etching layers on rail treads. Wear 191, 133-140.

Chrysochoos, A., Licht, C., Peyroux, R., 2003. A one-dimensional thermomechanical modeling of phase change front propagation in a SMA monocrystal. Comptes Rendus Mécanique 331, 25-32.

Eleöd, A., Oucherif, F., Devecz, J., Berthier, Y., 1999, Conception of numerical and experimental tools for study of the Tribological Transformation of Surface (TTS). Proceedings of Lubrication at the Frontier: The Role of the Interface and Surface Layers in the Thin Film and Boundary Regime, 673-682.

Garrigues, J., 2007. Fondements de la mécanique des milieux continus. Hermès Science Publications, France.

Greenwood, G.W., Johnson, R.H., 1965. The deformation of metals under small stresses during phase transformations. Proceedings of the Royal Society of London, series A: Mathematical and Physique Sciences 293, 403-422.

Leblond, J.B., Devaux, J., Devaux, J.C., 1989a. Mathematical modelling of transformation plasticity in steels - Case of ideal-plastic phases. International Journal of Plasticity 5, 551-571.

Leblond, J.B., Devaux, J., Devaux, J.C., 1989b. Mathematical modelling of transformation plasticity in steels - Coupling with strain hardening phenomena. International Journal of Plasticity 5, 573-591.

Liu, S.B., Wang, Q., 2003. Transient Thermoelastic Stress Fields in a HalfSpace. ASME Journal of Tribology 125, 33-43.

Nguyen, Q.S., 1977. On the elastic-plastic initial-boundary value problem and its numerical integration. International Journal for Numerical Methods in Engineering 11, 817-832.

Österle, W., Rooch, H., Pyzalla, A., Wang, L., 2001. Investigation of white etching layers on rails by optical microscopy, electron microscopy, Xray and synchrotron X-ray diffraction. Materials Science and Engineering: A 303, 150-157.

Sato, Y., Matsumoto, A., Knothe, K., 2002. Review on rail corrugation studies. Wear 253, 130-139.

Simo, J.C., Taylor, R.L., 1985. Consistent tangent operators for rateindependent elastoplasticity. Computer Methods in Applied Mechanics and Engineering 48, 101-118.

Taleb, L., Sidoroff, F., 2003. A micromechanical modeling of the Greenwood-Johnson mechanism in transformation induced plasticity. International Journal of Plasticity 19, 1821-1842.

Van, K.D., Maitournam, M.H., 1994, Thermomechanical state near rolling contact area. Proceedings of the 20th LeedsLyon Symposium, Dissipative Processes in Tribology, 423-428.

Van, K.D., Maitournam, M.H., Prasil, B., 1996. Elastoplastic analysis of repeated moving contact - Application to railways damage phenomena. Wear 196, 77-81. 